\section{Sudden Death in a Young Asthmatic}

SIR,-I would like to congratulate Dr. Ian Gregg and Dr. John Batten (5 April, p. 29) on publishing the details of a 14-year-old child who died of asthma.

Previous reports of death in asthma ${ }^{12}$ have laid emphasis on the high risk of death in this age group and have indicated that the main cause of death may be abuse of pressurized bronchodilator aerosols.

The paper of Gregg and Batten draws attention to the faot that underprescription of steroids may be dangerous, particularly in patients who have received previous steroid therapy. The importance of this case report lies in the warning against steroid withdrawal in the presence of considerably reduced respiratory function in those patients who are known to record normal results when on adequate steroid therapy. The authors mention that disodium cromoglycate may reduce the symptoms of asthma without a concurrent increase in respiratory function. Disodium cromoglycate is a valuable new addition to the treatment of asthma, particularly in acute allergic episodes, but it should be known that it may be possible for it on occasion to mask the warning symptoms of impending status asthmaticus.

The report of this case was particularly interesting in that there was post-mortem evidence of widespread mucous plugging of the medium airways; there was evidence that these mucous plugs had been present for several days before death and that during this time strenuous exercise had been taken, which must have led to considerable hypoxua. This report emphasizes that sudden and unexpected death in asthma may occur in the virtual absence of bronchodilator therapy-a fact which has tended to be overlooked in the light of publicity given to overprescription of bronchodilators.

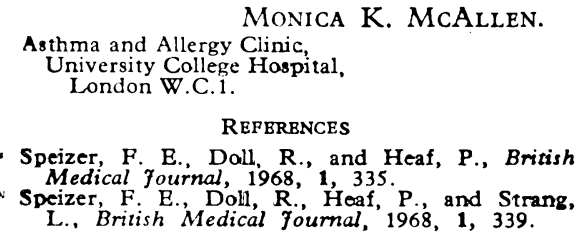

SIR,-This sad loss of a young boy reported by Dr. Ian Gregg and Dr. John Batten (5 April, p. 29) calls for a sober review of all the circumstances of the occurrence. The authors deserve full credit for publishing this case in detail, because this is the only way we can learn how to treat such patients. We read that the cough had been continuous (a sure sign that the cough was a strong contributory factor to the asthma), and that the severity of the case had been recognized-otherwise he would not have been treated with high doses of prednisone. This was started on 19 March with $30 \mathrm{mg}$./ day and reduced at frequent intervals over a period of 19 days. Each reduction would therefore have been made about every four deys. Potassium iodide was apparently not given.

If such patients die they do so invariably -as happened here-with (and probably from) mucous plugging of their bronchi. There are only two ways of reducing this hypersecretion-namely, potassium iodide and corticosteroids. The former, in optimal dosage, acts within 12 to 24 hours; the latter reduces hypersecretion within only two to three weeks. In my department every patient with viscous hypersecretion is given potassium iodide, often with immediate improvement. In severe cases like this prednisone is also necessary but ito initial high dose is not reduced quickly; when the patient has reached $15 \mathrm{mg} . /$ day he is left on this for one or two weeks until his hypersecretion is controlled. Only then is it considered wise to go down to the maintenance dose which is reached within another three to four weeks in small stages.

It is with extreme reluctance that I put forward these points because it is easy to be wise after the event, and because what has happened here could have happened to myself or anybody else. But I wonder why potassium iodide (which would reduce sputum viscosity effectively) is not considered more seriously as a valuable asthma remedy, since it has been used since the time of Strümpell. ${ }^{1}$ Our recorded experience with this substance extends now to over 2,500 patients. ${ }^{2}$ The history of the patient described by Dr. Gregg and Dr. Batten also shows that little importance should be attached to the often-observed subjective wellbeing of the patient after recovery from a severe asthmatic state. We know that in spite of this their $\mathrm{PaO}_{2}$ may remain subnormal for weeks and that they are therefore still very vulnerable. In these circumstances I cannot see any clear evidence that disodium cromoglycate, a drug which in my experiences helps only a small minority of patients, has contributed to the sense of wellbeing and so deprived the patient of warning symptoms.

$$
\begin{aligned}
& \text { Berlin } 65, \\
& \text { West Germany. }
\end{aligned}
$$

Strümpell, A., Lehrbuch der Speciellen Pathologie und Therapie, 1884. Leipzig, Vogel.

- Hercheimer H., and Bewersdorf, H., British Medical fournal, $1969,2,220$

\section{Weight Reduction in Obesity}

SIR,- We read with interest the article by Dr. J. F. Munro and others (5 April, p. 13). Sustained loss of weight has been reported in obese non-diabetic patients during treatment both with fenfluramine ${ }^{1}$ and with biguanides, ${ }^{2}$ without any attempt to impose a dietary regimen. We have recently completed a double-blind crossover trial comparing the efficacy of fenfluramine with metformin used at two dose levels in promoting weight reduction in a group of 34 obese women, but combined with dietary advice. Apart from obesity these women were in good health. In each case diabetes mellitus and abnormalities of thyroid function were excluded. The patients had all been obese for many years and had previously attempted weight reduction by dieting under medical supervision without success.

The study was conducted over four consecutive periods of eight weeks. Throughout the whole period of 32 weeks each patient was advised to take a diet providing 1,000 calories daily. The four treatment regimens studied were: (1) Control tablets containing inert material ; (2) metformin 0.5 g. thrice daily;
(3) metformin $1.0 \mathrm{~g}$. thrice daily; and (4) fenfluramine $26.6 \mathrm{mg}$. thrice daily. Identical tablets were prepared and each compound was given to every patient for eight weeks. The order of administration of the drugs was randomized using a Latin square technique, excep that the period of treatment with the larger dose of metformin always followed treatment with the smaller dose. Measurements of body weigh and skinfold thickness on the mid-triceps and subscapular areas were made at intervals of two weeks during the 32 weeks of the study.

Fenfluramine in a daily dose of $80 \mathrm{mg}$ was more effective than control tablets $(P<$ 0.001 ), and achieved almost the same mean total weight loss in eight weeks $(5.20 \pm 0.63$ kg.: mean \pm standard error) as the two regimens of metformin added together over 16 weeks $(5.61 \pm 0.55 \mathrm{~kg}$.). The effect of fenfluramine was still evident even during the fourth period of eight weeks. In contrast, the effect of metformin declined over the 16 weeks of treatment, despite doubling the dose during the second period of eight week of treatment, and by the fourth period of the whole trial there was no significant difference from control tablets.

On the other hand, reductions in skinfold thickness were sustained throughout the four periods with fenfluramine and metformin, suggesting that both compounds were having a metabolic effect on subcutaneous fat which was not necessarily apparent from changes in body weight. We observed side-effects with metformin similar to those described by Dr. Munro and his colleagues, and felt that these were of sufficient severity to make this drug unsuitable for the treatment of non-diabetic obese patients, especially as it was so considerably less effective than fenfluramine. The side-effects with fenfluramine were mild and did not interfere with the management of these patients. A full report on this clinical trial will appear elsewhere. . -We are, etc.,

A. A. H. LAWSON

A. M. C. GIBSON. P. Peattie.

Department of Medicine,
Western General Hospital Western General
Edinburgh 4.

REFERENCES

1. Lambusier, P., Presse Médicale, 1963, 4, 181. and Forsham, P. H., Metabolism, 1963, 12 . 278 .

Lawson, A. A. H., and Strong, J. A., Proceeding of an International Symposium on Amphetapress.

\section{Adverse Non-drug Reactions}

SiR,-Y Your leading article "Adverse Nondrug Reactions" (29 March, p. 798) draws attention to certain myths in diagnostic nomenclature which perhaps have never been serious problems for the less academically inclined clinician. In the three examples given-namely, thyroid, calcium, and iron "pseudo-deficiencies"-the range of symptoms mentioned is similar. In all lethargy is found, either as such, or as tiredness; also in all effects even more directly related to the psyche are reported-nervousness, mental symptoms or, more specifically, irritability. To the less esoteric clinician these would be the cases of debility, general or nervous, of earlier decades, some based on psychodynamic mechanisms and reactions to stresses. 